\title{
Modeling Length of Hospital Stay for Patients With COVID-19 in West Sumatra Using Quantile Regression
}

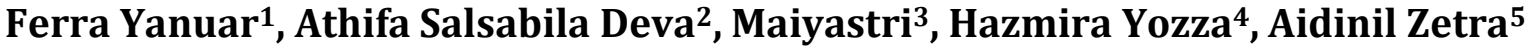 \\ 1,2,3,4Mathematics Department, Faculty of Mathematics and Natural Sciences, \\ Universitas Andalas, Padang \\ ${ }^{5}$ Political Sciences Department, Faculty of Social and Political Sciences, \\ Universitas Andalas, Padang \\ Email: ferrayanuar@sci.unand.ac.id, athifasalsabila4300@gmail.com, \\ maiyastri@sci.unand.ac.id, hazmirayozza@sci.unand.ac.id, aidinil@soc.unand.ac.id
}

\begin{abstract}
This study aims to construct the model for the length of hospital stay for patients with COVID-19 using quantile regression and Bayesian quantile approaches. The quantile regression models the relationship at any point of the conditional distribution of the dependent variable on several independent variables. The Bayesian quantile regression combines the concept of quantile analysis into the Bayesian approach. In the Bayesian approach, the Asymmetric Laplace Distribution (ALD) distribution is used to form the likelihood function as the basis for formulating the posterior distribution. All 688 patients with COVID-19 treated in M. Djamil Hospital and Universitas Andalas Hospital in Padang City between March-July 2020 were used in this study. This study found that the Bayesian quantile regression method results in a smaller 95\% confidence interval and higher value than the quantile regression method. It is concluded that the Bayesian quantile regression method tends to yield a better model than the quantile method. Based on the Bayesian quantile regression method, it was found that the length of hospital stay for patients with COVID-19 in West Sumatra was significantly influenced by Age, Diagnoses, and Discharge status.
\end{abstract}

Keywords: Length of hospital stay; Bayesian quantile regression; Asymmetric Laplace Distribution (ALD)

\section{INTRODUCTION}

The problem of COVID-19 has become the concern of the world community from every group. In cases of being infected with COVID-19 in West Sumatra Province, not a few people have been declared cured, died, or are undergoing treatment at the hospital. People with criteria for severe symptoms of COVID-19 must undergo treatment in a hospital [1]. Certain factors influence the length of stay of COVID-19 patients. An estimation of the regression model parameters is carried out using quantile regression and Bayesian quantile regression methods to identify the factors that influence the length of stay of COVID-19 patients. The estimated length of stay for COVID-19 patients who are hospitalized can be used for specific purposes such as in health service activities. the need for health facilities at each level of health care. and the preparation of decisions related to mitigation scenarios and preparedness for COVID-19 [2]-[4]. 
If linear model assumptions are fulfilled, such as no multicollinearity, homoscedasticity, and no autocorrelation, the Ordinary Least Squares (OLS) method is used to estimate the model parameters [5]. In the preliminary analysis, data on the length of stay of COVID-19 patients in West Sumatra Province were not normally distributed. Therefore, the use of OLS was not efficient in estimating model parameters. For this reason, an analysis of the estimated parameters was carried out using quantile regression and Bayesian quantile regression. Quantile regression analysis was chosen because in estimating the parameters, it does not require any assumptions, including the assumption of normality, which only requires large data. The merging of quantile analysis into Bayesian concepts is carried out so that the resulting estimator becomes more effective and natural so that it can produce a better predictive model that is closer to the actual value [6], [7].

Research related to Bayesian quantile regression was initiated by $\mathrm{Yu}$ and Mooyed [8]. Research on this topic then developed rapidly, including research on numerical simulations in estimating the parameters of the Bayesian quantile regression method using the Gibbs sampling algorithm [9]. The application of the Bayesian quantile regression method is also applied in the use of binary response data based on the Asymmetric Laplace Distribution (ALD) distribution [10]. Subsequent research discussed the analysis of variable selection in quantile regression using the Gibbs sampling concept [7]. Further Bayesian quantile regression analysis was also used to estimate the model by approximating the likelihood function [11], as well as the analysis of posterior inference with the likelihood of the ALD distribution [12]. The application of Bayesian quantile regression was also used in modeling the Jeonse deposit in Korea [13]. Oh et al. do selecting variables using the Bayesian quantile regression method using the SavageDickey density ratio [14]. Furthermore, the application of Bayesian quantile regression was also applied in constructing a low birth weight model using the Gibbs Sampling algorithm approach [15].

This study aims to construct a model of length of stay for COVID-19 patients using quantile and Bayesian quantile regression methods to then compare the results between two methods. This case is important to be investigated since the cases of Covid-19 is increasing. As the results, rooms in hospitals become full. For this reason, this research needs to be carried out in an effort to find out what factors affect the length of stay of COVID-19 patients. This research will give information on how to shorten the length of stay of COVID-19 patients.

\section{METHODS}

\section{Material}

Huskamp et al. and Kaufman et al. have found that mortalities are higher for the old populace than young populace [16], [17]. Yuki et al. recognized that older patients were more powerless to longer the length of hospital stay than younger patients [18]. This information implies that age could influence the length of hospital stay of a patient. Many studies also investigated that the presence of hypertension, diabetes, and coronary artery disease were considered as hazard factors to Covid-19 [19]. Gebhard et al, demonstrated that Covid-19 is deadlier for infected men than women [20].

The hypothesis model is constructed based on literatures to be then fitted to the data. The data used were 688 COVID-19 patients treated at M. Djamil Hospital, Padang City, and Andalas University Hospital in March-July 2020. In this study, the variables used are factors that are assumed to affect the length of stay of COVID-19 patients in West Sumatra 
Province, they are: Age $\left(X_{1}\right)$, Gender $\left(X_{2}\right)$ with male and female categories, Diagnosis of COVID-19 $\left(X_{3}\right)$ by categories are Asymptomatic Person (Asymp), Person Under Supervision (PerUS), Patients Under Supervision (PaUS), and Positive, Discharge status $\left(X_{4}\right)$ with the categories are recovered, died, forced discharge, outpatient, referred to another hospital, and the number of Comorbid $\left(X_{5}\right)$. Table 1 presents the frequency distribution for data of COVID-19 patients by categorical independent variables, i.e., Gender, Diagnosis, and Discharge status. Table 1 shows that most Diagnose of the respondents are PaUS (Patients Under Supervision) with $87.7 \%$ of all respondents and $73.3 \%$ respondents were Recovered.

Table 1. Frequency Distribution of COVID-19 Patients for Categorical Independent Variables

\begin{tabular}{cccc}
\hline Variable & Category & Frequency & Percentage \\
\hline \multirow{2}{*}{ Gender $\left(X_{1}\right)$} & Male & 347 & 50.4 \\
& Female & 341 & 49.6 \\
& Asymp & 1 & 0.1 \\
Diagnose $\left(X_{3}\right)$ & PerUS & 6 & 0.9 \\
& PaUS & 604 & 87.8 \\
& Positive & 77 & 11.2 \\
Discharge Status & Recovered & 504 & 73.3 \\
$\left(X_{4}\right)$ & Died & 141 & 20.5 \\
& Forced discharge & 30 & 4.4 \\
& Outpatient & 4 & 0.6 \\
\hline
\end{tabular}

In Figure 1 below, part (a) shows that the length of stay for COVID-19 patients has a histogram that is skewed to the left, while part (b) shows that some data are not located around a linear line. Based on both figures, these are informed that the data on the length of stay of COVID-19 patients is not normally distributed.

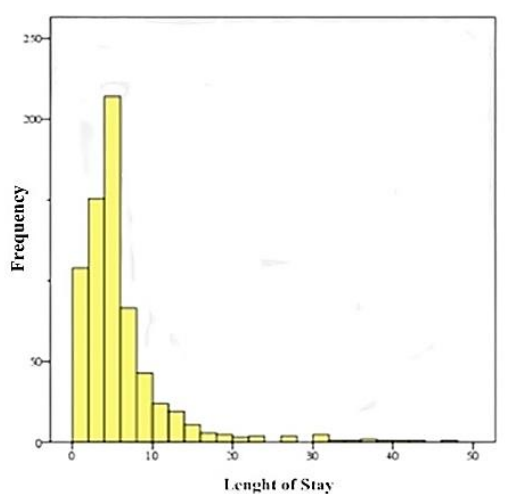

(a)

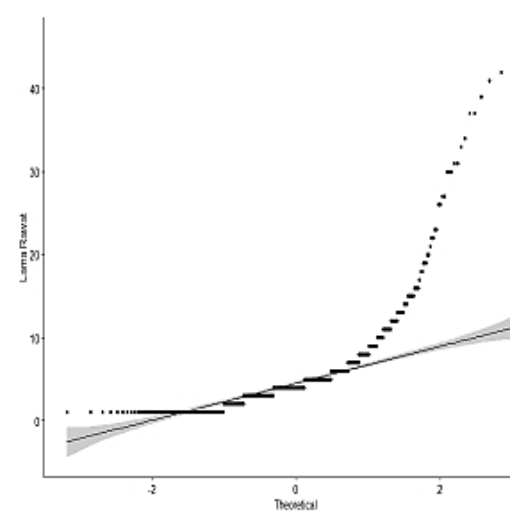

(b)

Figure 1. Data of Length of Hospital Stay: (a) Histogram and (b) QQ-Plot

\section{Quantile Regression Method}

Assummed that $\boldsymbol{y}=\left(y_{1}, y_{2}, \cdots, y_{n}\right)^{\prime}$ is response variable vector and $\boldsymbol{x}=\left(x_{1}, x_{2}, \cdots, x_{k}\right)^{\prime}$ is a covariate vector. In general, a linear regression equation model for the $\tau$-th quantile. 
where $0<\tau<1$ with $n$ sample and $k$ predictor for $i=1,2, \ldots, n$ written in the form:

$y_{i}=\beta_{0 \tau}+\beta_{1 \tau} x_{i 1}+\beta_{2 \tau} x_{i 2}+\cdots+\beta_{k \tau} x_{i k}+\varepsilon_{i}$,

where $\boldsymbol{\beta}(\tau)$ is parameter's vector and $\boldsymbol{\varepsilon}$ is the leftover vector.

The $\tau$-th conditional quantile function in the quantile regression method is defined as $Q_{y_{i}}\left(\tau \mid x_{i}\right)=x^{\prime}{ }_{i} \boldsymbol{\beta}(\tau)$ then the estimated value of the parameter is $\widehat{\boldsymbol{\beta}}(\tau)$ obtained by minimizing [21]:

$\sum_{i=1}^{n} \rho_{\tau}\left(y_{i}-x_{i}^{\prime} \boldsymbol{\beta}\right)$

where $\rho_{\tau}(u)=u(\tau-I(u<0))$ is a loss function which is equivalent to :

$$
\rho_{\tau}(\varepsilon)=\varepsilon(\tau I(\varepsilon>0)-(1-\tau) I(\varepsilon<0)),
$$

$I($.$) is an indicator function. with value 1$ if $I($.$) is true and zero rest.$

Minimization of Equation (2) was done by using the simplex method in linear programming. However, using the simplex method in estimating parameters is complicated to do. Therefore, an approach with the Bayes method is carried out so that the parameter estimation process becomes a little easier.

\section{Bayesian Quantile Regression Method}

$\mathrm{Yu}$ and Mooyed [8] found that minimizing the loss function of the quantile regression is equivalent to maximizing the likelihood function formed from the data assumed to be distributed in the Asymmetric Laplace Distribution (ALD). The ALD is used in the likelihood distribution to make Bayesian estimators more effective and natural. This estimation resulted in the ALD distribution is a possible parametric relationship between the minimization problem of Equation (2) and the maximum likelihood theory [7]. In addition, the quantile regression loss function is identical to the likelihood function of ALD [22].

The ALD distribution is one of the continuous probability distributions. A random variable $\varepsilon$ has an ALD distribution with probability density function $f(\varepsilon)$ [7], [8]:

$$
f_{\tau}(\varepsilon)=\tau(1-\tau) \exp \left(-\rho_{\tau}(\varepsilon)\right) \text {. }
$$

where $0<\tau<1$ and $\rho_{\tau}(\varepsilon)$ where defined in Equation (3).

The estimation of model parameters using the Bayesian quantile regression method can be done for any data distribution by assuming the following [8]:

1. $f\left(y ; \mu_{i}\right)$ has ALD distribution.

2. $g\left(\mu_{i}\right)=x_{i}^{\prime} \boldsymbol{\beta}(\tau)$.

The observation was given by $\boldsymbol{y}=\left(y_{1}, y_{2}, \cdots, y_{n}\right)$. Based on Equation (4), to combine the quantile regression method into the Bayesian method to estimate the parameter, $\boldsymbol{\beta}$. ALD was used to form the likelihood function. The ALD has a combined representation of several distributions based on the exponential distribution and normal distribution [9]. A random variable $\varepsilon$ can be expressed in:

$$
\varepsilon=\theta z+p u \sqrt{z},
$$

where $\theta=\frac{1-2 \tau}{(1-\tau) \tau}$ and $p^{2}=\frac{2}{(1-\tau) \tau}$.

The $\tau$-th quantile regression model can be written as:

$$
y_{i}=x_{i}^{\prime} \boldsymbol{\beta}_{\tau}+\sigma \theta z_{i}+\sigma p u_{i} \sqrt{z_{i}},
$$

where $z_{i} \sim \exp (1)$ and $u_{i} \sim N(0,1), v_{i}=\sigma z_{i}, \quad \boldsymbol{v}=\left(v_{1}, v_{2}, \cdots, v_{n}\right)^{\prime}$. Because of $z_{i} \sim \exp (1)$ then $v_{i} \sim \exp (\sigma)$, and $i=1,2, \cdots, n$. So, we get the probability density function of $y_{i}$ :

$$
f\left(y_{i} ; \boldsymbol{\beta}_{\tau}, v_{i}, \sigma\right)=\frac{1}{p \sqrt{\sigma v_{i}} \sqrt{2 \pi}} \exp \left(-\frac{\left(y_{i}-\left(x_{i}^{\prime} \boldsymbol{\beta}_{\tau}+\theta v_{i}\right)\right)^{2}}{2 p^{2} \sigma v_{i}}\right),
$$

and the likelihood function is obtained as follows: 


$$
L\left(\boldsymbol{\beta}_{\tau}, \boldsymbol{v}, \sigma\right) \propto\left(\prod_{i=1}^{n}\left(\sigma v_{i}\right)^{-\frac{1}{2}}\right)\left(\exp \left(-\sum_{i=1}^{n} \frac{\left(y_{i}-\left(x_{i}^{\prime} \boldsymbol{\beta}_{\tau}+\theta v_{i}\right)\right)^{2}}{2 p^{2} \sigma v_{i}}\right)\right) .
$$

Then, the prior distribution is selected for the parameter $\boldsymbol{\beta}_{\tau} \sim N\left(b_{0}, \boldsymbol{B}_{0}\right)$. $v_{i} \sim \exp (\sigma)$, and $\sigma \sim I G(a, b)$. The posterior distribution is obtained, i.e:

$$
\begin{aligned}
&\left(\boldsymbol{\beta}_{\tau} \mid \boldsymbol{v}, \sigma, \boldsymbol{y}\right) \sim N {\left[( \boldsymbol { B } _ { 0 } { } ^ { - 1 } + x _ { i } ( p ^ { 2 } \sigma \boldsymbol { v } ) ^ { - \mathbf { 1 } } x _ { i } ^ { \prime } ) ^ { - 1 } \left(\boldsymbol{B}_{0}{ }^{-1} b_{\mathbf{0}}+x_{i}\left(p^{2} \sigma \boldsymbol{v}\right)^{-\mathbf{1}} \boldsymbol{y}\right.\right.} \\
&\left.\left.-x_{i}\left(p^{2} \sigma \boldsymbol{v}\right)^{-\mathbf{1}} \theta \boldsymbol{v}\right),\left(\boldsymbol{B}_{0}{ }^{-1}+x_{i}\left(p^{2} \sigma \boldsymbol{v}\right)^{-\mathbf{1}} x_{i}^{\prime}\right)^{-1}\right] ; \\
&\left(v_{\boldsymbol{i}} \mid \boldsymbol{\beta}_{\tau}, \sigma, \boldsymbol{y}\right) \sim G I G\left(\frac{1}{2},\left(\frac{\left(y_{i}-x_{i}^{\prime} \boldsymbol{\beta}_{\tau}\right)^{2}}{p^{2} \sigma}\right),\left(\frac{2}{\sigma}+\frac{\theta^{2}}{p^{2} \sigma}\right)\right) ; \\
&\left(\sigma \mid \boldsymbol{\beta}_{\tau}, \boldsymbol{v}, \boldsymbol{y}\right) \sim I G\left(\left(a+\frac{3 n}{2}\right),\left(b+\sum_{i=1}^{n} v_{i}+\sum_{i=1}^{n} \frac{\left(y_{i}-\left(x_{i}^{\prime} \boldsymbol{\beta}_{\tau}+\theta v_{i}\right)\right)^{2}}{2 p^{2} v_{i}}\right)\right) .
\end{aligned}
$$

These posterior distribution then are used to estimate mean posterior and variance posterior as point estimate for unknown parameter using Gibbs sampling iteration method [23], [24].

The goodness of fit for both methods is measured using Pseudo $R^{2}$ [25]. The formula for Pseudo $R^{2}$ is as follows:

$$
\text { Pseudo } R^{2}=1-\frac{R A S W_{\tau}}{T A S W_{\tau}},
$$

where $R A S W_{\tau}$ is the residual absolute sum of weighted differences between the observed dependent variable and the estimated quantile of conditional distribution in the more complex model. While, $T A S W_{\tau}$ is the total absolute sum of weighted differences between the observed dependent variable and the estimated quantile of conditional distribution in the simplest model. The range values for Pseudo $R^{2}$ are between zero and one. The value of Pseudo $R^{2}$ indicates the goodness of fit of the proposed model in explaining the variance of the response variable. The higher the value of Pseudo $R^{2}$ the better the proposed model obtained.

\section{RESULTS AND DISCUSSION}

Data analysis begins with fitting the data to the hypothesis model using the OLS method to select the significant variables involved for modeling in the quantile and Bayesian analysis. Based on OLS analysis, the variables of Age, Diagnosis, and Discharge status contributed significantly. Furthermore, a model of the length of stay for COVID-19 patients is constructed using the quantile regression method and the Bayesian quantile regression method. The analysis results are then compared between both methods by looking at the width of the $95 \%$ confidence interval and Pseudo $R^{2}$ of the selected quantile. The quantile used are $0.10 ; 0.25 ; 0.50 ; 0.75$; dan 0.90 . R software was used to analyze the data. The results of the analysis from both methods are provided in Table 3 . 
Table 3. Comparison between Quantile and Bayesian Quantile Method

\begin{tabular}{|c|c|c|c|c|}
\hline \multirow{2}{*}{ Indicator Variables } & \multicolumn{2}{|c|}{ Quantile Method } & \multicolumn{2}{|c|}{ Bayesian Quantile Method } \\
\hline & Estimate & $95 \% \mathrm{CI}$ & Estimate & $95 \%$ CI \\
\hline \multicolumn{5}{|l|}{$\tau=0.10$} \\
\hline Intersep & 2.0000 & NA & -1.5006 & 14.6799 \\
\hline Age $\left(X_{1}\right)$ & 0.0000 & 0.0000 & 0.0002 & 0.0041 \\
\hline \multicolumn{5}{|l|}{ Diagnose $\left(X_{3}\right)$} \\
\hline PerUS $\left(X_{3 D 1}\right)$ & -2.0000 & NA & 1.3864 & 15.3326 \\
\hline $\operatorname{PaUS}\left(X_{3 D 2}\right)$ & -1.0000 & NA & 2.4139 & 14.6589 \\
\hline Positive $\left(X_{3 D 3}\right)$ & 0.0000 & NA & 3.1337 & 14.8359 \\
\hline \multicolumn{5}{|l|}{ Discharge Status $\left(X_{4}\right)$} \\
\hline Recovered $\left(X_{4 D 1}\right)$ & $2.0000^{*}$ & 0.0000 & $2.0446^{*}$ & 0.6021 \\
\hline $\operatorname{Died}\left(X_{4 D 2}\right)$ & 0.0000 & 0.0000 & 0.0275 & 0.6264 \\
\hline Outpatient $\left(X_{4 D 3}\right)$ & 0.0000 & NA & -0.2693 & 5.0697 \\
\hline $\begin{array}{l}\text { Referred to another } \\
\text { hospital }\left(X_{4 D 4}\right)\end{array}$ & 0.0000 & NA & -0.1649 & 2.0374 \\
\hline \multicolumn{5}{|l|}{$\tau=0.25$} \\
\hline Intersep & 1.0000 & NA & -1.0118 & 14.2889 \\
\hline Age $\left(X_{1}\right)$ & 0.0000 & 0.0000 & 0.0014 & 0.0094 \\
\hline \multicolumn{5}{|l|}{ Diagnose $\left(X_{3}\right)$} \\
\hline PerUS $\left(X_{3 D 1}\right)$ & -1.0000 & NA & 1.2671 & 14.8492 \\
\hline $\operatorname{PaUS}\left(X_{3 D 2}\right)$ & 0.0000 & NA & 2.1446 & 14.2644 \\
\hline Positive $\left(X_{3 D 3}\right)$ & 3.0000 & NA & $5.1174^{*}$ & 14.4329 \\
\hline \multicolumn{5}{|l|}{ Discharge Status $\left(X_{4}\right)$} \\
\hline Recovered $\left(X_{4 D 1}\right)$ & $3.0000^{*}$ & 1.2544 & $2.6699^{*}$ & 1.2514 \\
\hline $\operatorname{Died}\left(X_{4 D 2}\right)$ & 0.0000 & 0.9709 & -0.2344 & 1.1873 \\
\hline Outpatient $\left(X_{4 D 3}\right)$ & 0.0000 & NA & 1.5336 & 6.3011 \\
\hline $\begin{array}{l}\text { Referred to another } \\
\text { hospital }\left(X_{4 D 4}\right)\end{array}$ & 0.0000 & 1.1155 & 0.1684 & 2.9599 \\
\hline \multicolumn{5}{|l|}{$\tau=0.50$} \\
\hline Intersep & 1.0000 & NA & 1.4877 & 15.5613 \\
\hline Age $\left(X_{1}\right)$ & 0.0000 & 0.0000 & 0.0002 & 0.0010 \\
\hline \multicolumn{5}{|l|}{ Diagnose $\left(X_{3}\right)$} \\
\hline $\operatorname{PerUS}\left(X_{3 D 1}\right)$ & 1.0000 & NA & 0.9143 & 16.1529 \\
\hline $\operatorname{PaUS}\left(X_{3 D 2}\right)$ & 1.0000 & NA & 0.9518 & 15.4168 \\
\hline Positive $\left(X_{3 D 3}\right)$ & 7.0000 & NA & 6.8087 & 15.6258 \\
\hline \multicolumn{5}{|l|}{ Discharge Status $\left(X_{4}\right)$} \\
\hline Recovered $\left(X_{4 D 1}\right)$ & $3.0000^{*}$ & 1.3265 & $2.4889^{*}$ & 2.1480 \\
\hline Died $\left(X_{4 D 2}\right)$ & $-1.0000^{*}$ & 2.1006 & $-1.3962^{*}$ & 2.1555 \\
\hline Outpatient $\left(X_{4 D 3}\right)$ & 3.0000 & 6.1705 & 2.4900 & 7.1299 \\
\hline $\begin{array}{l}\text { Referred to another } \\
\text { hospital }\left(X_{4 D 4}\right)\end{array}$ & 0.0000 & 2.9157 & 0.0397 & 4.2175 \\
\hline \multicolumn{5}{|l|}{$\tau=0.75$} \\
\hline Intersep & 2.0000 & NA & 5.7668 & 22.7172 \\
\hline Age $\left(X_{1}\right)$ & $-1.05 \times 10^{-7}$ & 0.0210 & $-0.0071^{*}$ & 0.0229 \\
\hline \multicolumn{5}{|l|}{ Diagnose $\left(X_{3}\right)$} \\
\hline PerUS $\left(X_{3 D 1}\right)$ & 5.0000 & NA & 0.1273 & 24.1500 \\
\hline $\operatorname{PaUS}\left(X_{3 D 2}\right)$ & 2.0000 & NA & -1.4964 & 22.7243 \\
\hline Positive $\left(X_{3 D 3}\right)$ & 12.0000 & NA & 9.2450 & 23.1280 \\
\hline
\end{tabular}




\begin{tabular}{|c|c|c|c|c|}
\hline \multirow{2}{*}{ Indicator Variables } & \multicolumn{2}{|c|}{ Quantile Method } & \multicolumn{2}{|c|}{ Bayesian Quantile Method } \\
\hline & Estimate & $95 \% \mathrm{CI}$ & Estimate & $95 \%$ CI \\
\hline \multicolumn{5}{|l|}{ Discharge Status $\left(X_{4}\right)$} \\
\hline Recovered $\left(X_{4 D 1}\right)$ & $2.0000^{*}$ & 2.0233 & $2.2849^{*}$ & 2.2233 \\
\hline $\operatorname{Died}\left(X_{4 D 2}\right)$ & $-2.0000^{*}$ & 1.5733 & $-1.8134^{*}$ & 2.3198 \\
\hline Outpatient $\left(X_{4 D 3}\right)$ & 2.0000 & NA & 2.8668 & 8.5570 \\
\hline $\begin{array}{l}\text { Referred to another } \\
\text { hospital }\left(X_{4 D 4}\right)\end{array}$ & 0.0000 & 9.5010 & 0.5922 & 5.9888 \\
\hline \multicolumn{5}{|l|}{$\tau=0.90$} \\
\hline Intersep & -0.6596 & NA & 8.3752 & 36.4268 \\
\hline Age $\left(X_{1}\right)$ & -0.0213 & 0.0539 & $-0.0181^{*}$ & 0.0339 \\
\hline \multicolumn{5}{|l|}{ Diagnose $\left(X_{3}\right)$} \\
\hline PerUS $\left(X_{3 D 1}\right)$ & 8.7021 & NA & 0.2688 & 38.0224 \\
\hline PaUS $\left(X_{3 D 2}\right)$ & 5.6596 & NA & -2.9586 & 36.2285 \\
\hline Positive $\left(X_{3 D 3}\right)$ & 27.5957 & NA & 18.1682 & 37.1268 \\
\hline \multicolumn{5}{|l|}{ Discharge Status $\left(X_{4}\right)$} \\
\hline Recovered $\left(X_{4 D 1}\right)$ & $5.9362^{*}$ & 2.7045 & $5.2751^{*}$ & 2.7938 \\
\hline $\operatorname{Died}\left(X_{4 D 2}\right)$ & $-0.5957^{*}$ & 2.7368 & $-1.1434^{*}$ & 2.8253 \\
\hline Outpatient $\left(X_{4 D 3}\right)$ & 2.1702 & NA & 3.5015 & 10.9954 \\
\hline $\begin{array}{l}\text { Referred to another } \\
\text { hospital }\left(X_{4 D 4}\right)\end{array}$ & 0.7660 & NA & 1.2313 & 6.3550 \\
\hline
\end{tabular}

In Table 3, it can be seen that for the quantile regression method, the $X_{4 D 1}$ variable (Recovered) contributed significantly in each quantile, and the category Died is significant in the quantile $0.50 ; 0.75$; and 0.90 . Meanwhile, none were statistically significant for other categories in other quantiles in influencing the length of stay of COVID-19 patients.

Meanwhile, by using the Bayesian quantile regression method, the Age contributed significantly at the quantile 0.75 , and 0.90 in giving affects to the length of stay of COVID19 patients. While, diagnose variable (only Positive category) contributed significantly to the length of stay of COVID-19 patients in quantile 0.25, Discharge Status (only Recovered category) contributed significantly in all quantiles, Discharge Status (only Died category) is significant in quantile $0.50 ; 0.75$; and 0.90 to affect the length of stay of COVID-19 patients.

From the results of this estimation analysis, it is found that the Bayesian quantile regression method as a whole has more significant parameter and smaller 95\% confidence interval than the quantile regression method.

In order to determine the best method including the best model, it could be based on the higher value of Pseudo $R^{2}$. The Pseudo $R^{2}$ values for both methods for all selected quantiles are provided in Table 4.

Table 4. The Pseudo $R^{2}$ Values at All Selected Quantiles.

\begin{tabular}{|c|c|c|}
\hline \multirow{2}{*}{ Quantile $\tau^{t h}$} & \multicolumn{2}{|c|}{ Pseudo $R^{2}$} \\
\hline & Quantile & Bayesian Quantile \\
\hline 0.10 & 0.27030 & 0.27235 \\
\hline 0.25 & 0.57550 & 0.57843 \\
\hline 0.50 & 0.87950 & 0.88262 \\
\hline 0.75 & 0.93925 & 0.94244 \\
\hline 0.90 & 0.67508 & 0.67787 \\
\hline
\end{tabular}


In Table 4 above, it can be seen that for the quantile regression method, the model at quantile 0.75 is the best model because it has the highest value of Pseudo $R^{2}$, that is 0.93925 . This value informs that the proposed model can explain the variance of length of hospital stay for patients with COVID-19 is $93.925 \%$. This means that the proposed model at quantile 0.75 is acceptable and could be accepted. Meanwhile for the Bayesian quantile regression method, the quantile 0.75 is also as the best model because it has the highest value of Pseudo $R^{2}$, that is 0.94244 . This informs us that the model can explain the variance of the length of stay for COVID-19 patients by $94.244 \%$. Since the Pseudo $R^{2}$ value obtained from Bayesian quantile regression model is higher than quantile method at corresponding quantiles, we could conclude here that Bayesian quantile method tends to result better model than quantile method. Therefore, the best model for the length of stay of COVID-19 patients in West Sumatra is model at quantile 0.75 based on Bayesian quantile regression method. This proposed model is formulated as follows:

$$
\begin{gathered}
\hat{y}=5.7668- \\
-0.0071 X_{1}+0.1273 X_{3 D 1}-1.4964 X_{3 D 2}+9.2450 X_{3 D 3}+2.2849 X_{4 D 1} \\
-1.8134 X_{4 D 2}+2.8668 X_{4 D 3}+0.5922 X_{4 D 4} .
\end{gathered}
$$

There were $75 \%$ of the length of stay for COVID-19 patients diagnosed with PerUS (Person Under Supervision) is 0.1273 days longer than patients diagnosed with Asymp (Asymptotic Persons) assuming others constants. Around $75 \%$ of the length of stay for COVID-19 patients diagnosed with PaUS (Patients Under Supervision) is 1.4964 days longer than patients diagnosed with Asymp (Asymptotic Persons) assuming others constants. Approximately, 75\% of the length of stay of COVID-19 patients diagnosed with Positive was 9.2450 days longer than patients diagnosed with Asymp (Asymptotic Persons) assuming other variables constant. The similar interpretation could be stated for other variables.

Furthermore, the convergence test of the proposed parameter model obtained was carried out. Because of limited space, the selected results of these test are provided in Figure 2 below.

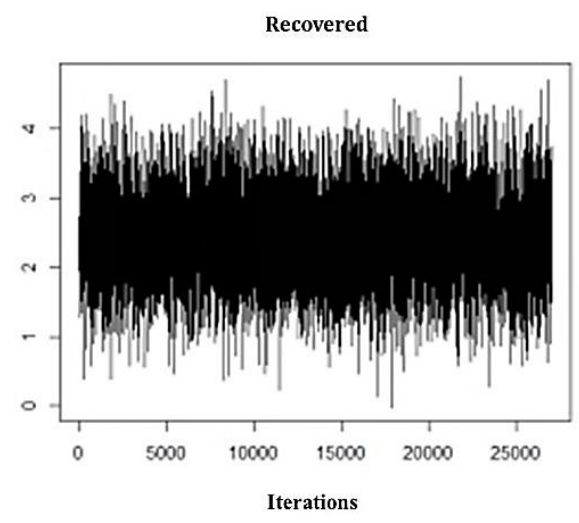

(a)

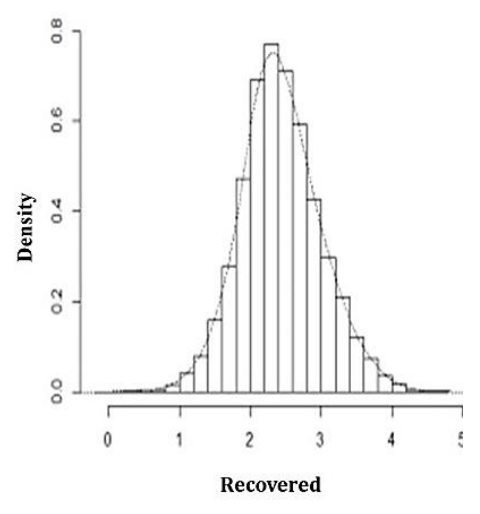

(b)

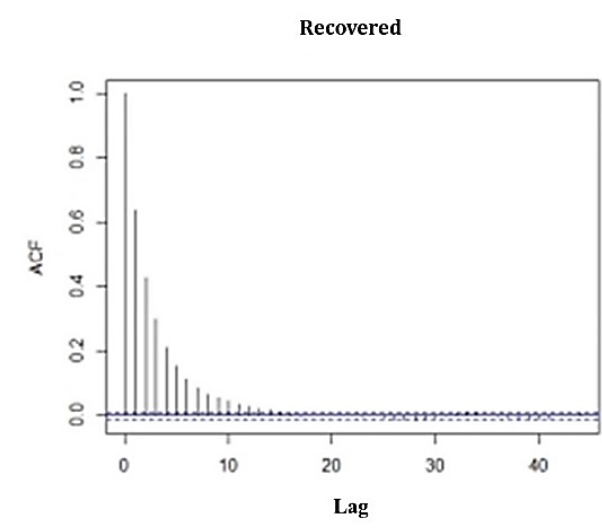

(c)

Figure 2. Convergency test for Category Recovered at quantile 0.75 (a) Trace-Plot, (b) DensityPlot, dan (c) ACF Plot

In Figure 2 (a), it can be seen that the resulting trace-plot forms a pattern that converges to a value so that it can be stated that the model parameters have converged. 
While in part (b), it can be seen that the resulting density plot resembles a normal distribution curve. It can be stated that the model parameters are normally distributed. Then in part (c), the resulting ACF plot shows a smaller autocorrelation value so that it can be stated that there is no autocorrelation between samples. Based on these convergency test, it can be concluded that the model parameters have converged and proposed model could be accepted.

\section{CONCLUSIONS}

This study found that the length of stay of COVID-19 patients in West Sumatra was influenced by Age, Diagnoses of COVID-19 patients, and Discharge status. From the analysis carried out, the Bayesian quantile regression method is better in modeling the length of stay of COVID-19 patients than quantile method. The $95 \%$ confidence interval based on Bayesian quantile regression is smaller, and the Pseudo $R^{2}$ value is greater than the quantile regression method.

\section{ACKNOWLEDGMENTS}

This research was funded by Directorate of Resources Directorate General of Higher Education, Ministry of Education, Culture, and Research and Technology of Indonesia, in accordance with Contract Number 104/E4.1/AK.04.PT/2021.

\section{REFERENCES}

[1] Kemenkes RI, "KMK No. HK.01.07-MENKES-413-2020 ttg Pedoman Pencegahan dan Pengendalian COVID-19.pdf." 2020. [Online]. Available: https://covid19.go.id/p/regulasi/keputusan-menteri-kesehatan-republikindonesia-nomor-hk0107menkes4132020

[2] N. Lapidus, X. Zhou, F. Carrat, B. Riou, Y. Zhao, and G. Hejblum, "Biased and unbiased estimation of the average length of stay in intensive care units in the Covid-19 pandemic," Ann. Intensive Care, vol. 10, no. 135, pp. 1-9, Dec. 2020, doi: 10.1186/s13613-020-00749-6.

[3] E. M. Rees et al., "COVID-19 length of hospital stay: a systematic review and data synthesis," BMC Med, vol. 18, no. 270, pp. 1-22, Dec. 2020, doi: 10.1186/s12916020-01726-3.

[4] S. Wu et al., "Understanding factors influencing the length of hospital stay among non-severe COVID-19 patients: A retrospective cohort study in a Fangcang shelter hospital," PLoS ONE, vol. 15, no. 10, p. e0240959, Oct. 2020, doi: 10.1371/journal.pone.0240959.

[5] N. Desviona and F. Yanuar, "Simulation Study of Autocorrelated Error Using Bayesian Quantile Regression," sci. technol. indones., vol. 5, no. 3, pp. 70-74, Jul. 2020, doi: 10.26554/sti.2020.5.3.70-74.

[6] R. Alhamzawi, K. Yu, and D. F. Benoit, "Bayesian adaptive Lasso quantile regression," Statistical Modelling, vol. 12, no. 3, pp. 279-297, Jun. 2012, doi: 10.1177/1471082X1101200304. 
[7] R. Alhamzawi and K. Yu, "Variable selection in quantile regression via Gibbs sampling," Journal of Applied Statistics, vol. 39, no. 4, pp. 799-813, Apr. 2012, doi: 10.1080/02664763.2011.620082.

[8] K. Yu and R. A. Moyeed, "Bayesian quantile regression," Statistics \& Probability Letters, vol. 54, pp. 437-447, 2001.

[9] H. Kozumi and G. Kobayashi, "Gibbs sampling methods for Bayesian quantile regression," Journal of Statistical Computation and Simulation, vol. 81, no. 11, pp. 1565-1578, Nov. 2011, doi: 10.1080/00949655.2010.496117.

[10] D. F. Benoit and D. Van den Poel, "Binary quantile regression: a Bayesian approach based on the asymmetric Laplace distribution," J. Appl. Econ., vol. 27, no. 7, pp. 1174-1188, Nov. 2012, doi: 10.1002/jae.1216.

[11] Y. Feng, Y. Chen, and X. He, "Bayesian quantile regression with approximate likelihood," Bernoulli, vol. 21, no. 2, pp. 832-850, May 2015, doi: 10.3150/13BEJ589.

[12] Y. Yang, H. J. Wang, and X. He, "Posterior Inference in Bayesian Quantile Regression with Asymmetric Laplace Likelihood: Bayesian Quantile Regression," International Statistical Review, vol. 84, no. 3, pp. 327-344, Dec. 2016, doi: 10.1111/insr.12114.

[13] E. J. Nam, E. K. Lee, and M.-S. Oh, "Bayesian quantile regression analysis of Korean Jeonse deposit," CSAM, vol. 25, no. 5, pp. 489-499, 2018, doi: 10.29220/CSAM.2018.25.5.489.

[14] M.-S. Oh, J. Choi, and E. S. Park, "Bayesian variable selection in quantile regression using the Savage-Dickey density ratio," Journal of the Korean Statistical Society, vol. 45, no. 3, pp. 466-476, 2016, doi: 10.1016/j.jkss.2016.01.006.

[15] F. Yanuar, A. Zetra, C. Muharisa, D. Devianto, A. R. Putri, and Y. Asdi, "Bayesian Quantile Regression Method to Construct the Low Birth Weight Model," J. Phys.: Conf. Ser., vol. 1245, p. 012044, Aug. 2019, doi: 10.1088/17426596/1245/1/012044.

[16] H. A. Huskamp, D. G. Stevenson, D. C. Grabowski, E. Brennan, and N. L. Keating, "Long and Short Hospice Stays among Nursing Home Residents at the End of Life," Journal of Palliative Medicine, vol. 13, no. 8, pp. 957-964, Aug. 2010, doi: 10.1089/jpm.2009.0387.

[17] B. G. Kaufman, C. A. Sueta, C. Chen, B. G. Windham, and S. C. Stearns, "Are Trends in Hospitalization Prior to Hospice Use Associated With Hospice Episode Characteristics?," Am J Hosp Palliat Care, vol. 34, no. 9, pp. 860-868, Nov. 2017, doi: $10.1177 / 1049909116659049$.

[18] K. Yuki, M. Fujiogi, and S. Koutsogiannaki, "COVID-19 pathophysiology: A review," Clinical Immunology, vol. 215, no. 108427, pp. 1-7, Jun. 2020, doi: 10.1016/j.clim.2020.108427.

[19] Y. Du et al., "Clinical Features of 85 Fatal Cases of COVID-19 from Wuhan. A Retrospective Observational Study," Am J Respir Crit Care Med, vol. 201, no. 11, pp. 1372-1379, Jun. 2020, doi: 10.1164/rccm.202003-05430C.

[20] C. Gebhard, V. Regitz-Zagrosek, H. K. Neuhauser, R. Morgan, and S. L. Klein, "Impact of sex and gender on COVID-19 outcomes in Europe," Biol Sex Differ, vol. 11, no. 1, pp. 1-13, Dec. 2020, doi: 10.1186/s13293-020-00304-9.

[21] R. Alhamzawi and K. Yu, "Conjugate priors and variable selection for Bayesian quantile regression," Computational Statistics \& Data Analysis, vol. 64, pp. 209-219, Aug. 2013, doi: 10.1016/j.csda.2012.01.014. 
[22] Y.Yang, H. J. Wang, and X. He, "Posterior Inference in Bayesian Quantile Regression with Asymmetric Laplace Likelihood: Bayesian Quantile Regression," International Statistical Review, vol. 84, no. 3, pp. 327-344, 2015, doi: 10.1111/insr.12114.

[23] C. Muharisa, F. Yanuar, and D. Devianto, "Simulation Study The Using of Bayesian Quantile Regression in Nonnormal Error," CAUCHY, vol. 5, no. 3, pp. 121-126, Dec. 2018, doi: 10.18860/ca.v5i3.5633.

[24] I. Ntzoufras, Bayesian modeling using WinBUGS. Hoboken, N.J: Wiley, 2009.

[25] F. Yanuar, H. Yozza, F. Firdawati, I. Rahmi, and A. Zetra, "Applying bootstrap quantile regression for the construction of a low birth weight model," Makara Journal of Health Research, vol. 23, no. 2, pp. 90-95, Aug. 2019, doi: 10.7454/msk.v23i2.9886. 